PAPER

\title{
Factors influencing PCR detection of viruses in cerebrospinal fluid of patients with suspected CNS infections
}

\author{
N W S Davies, L J Brown, J Gonde, D Irish*, R O Robinson, A V Swan, J Banatvala, R S Howard, \\ M K Sharief, P Muir**
}

See Editorial Commentary, p 10

J Neurol Neurosurg Psychiatry 2005;76:82-87. doi: 10.1136/jnnp.2004.045336

See end of article for authors' affiliations

.....................

Correspondence to: Dr Nicholas Davies, Department of Clinical Neurosciences, Hodgkin Building, Guy's Hospital, London SEI 1UL, UK: nicholas.davies@kcl.ac.uk

Received 10 May 2004

In revised form

8 August 2004

Accepted 9 August 2004
Background: Polymerase chain reaction (PCR) is used to detect viruses in the cerebrospinal fluid (CSF) of patients with neurological disease. However, data to assist its use or interpretation are limited.

Objective: We investigated factors possibly influencing viral detection in CSF by PCR, which will also help clinicians interpret positive and negative results.

Methods: CSF from patients with was tested for human herpesviruses types 1-6, JC virus, enteroviruses, and Toxoplasma gondii. The likelihood of central nervous system (CNS) infection was classified as likely, possible, or unlikely. PCR findings in these categories were compared using single variable and logistic regression analysis.

Results: Of 787 samples tested, 97 (12\%) were PCR positive for one or more viruses. Of episodes likely to be CNS viral infections, 30\% were PCR positive compared to $5 \%$ categorised as unlikely. The most frequent positive findings were Epstein Barr virus (EBV), enteroviruses, and herpes simplex virus (HSV). Enteroviruses and HSV were found predominantly in the likely CNS viral infection group, whereas EBV was found mainly in the unlikely group. Positive PCR results were more likely when there were 3-14 days between symptom onset and lumbar puncture, and when CSF white cell count was abnormal, although a normal CSF did not exclude a viral infection.

Conclusions: The diagnostic yield of PCR can be maximised by using sensitive assays to detect a range of pathogens in appropriately timed CSF samples. PCR results, in particular EBV, should be interpreted cautiously when symptoms cannot readily be attributed to the virus detected.
$\mathrm{T}$ he application of molecular biological techniques to routine clinical specimens has led to a revolution in diagnostic virology. The polymerase chain reaction (PCR) amplifies and therefore detects low copy numbers of specific nucleic acid sequences. Consequently, not only does PCR have a high sensitivity but also the ability to detect nonculture viable or fastidious organisms. ${ }^{1}$ Such techniques offer particular advantage in the diagnosis of central nervous system (CNS) viral infections where PCR has not only improved diagnostic sensitivity but also expanded the clinical phenotype of several virus infections. For example, it is now recognised that both herpes simplex type 2 (HSV-2) and varicella zoster virus (VZV) are common causes of aseptic meningitis in adults even without a rash. ${ }^{23}$

Prior to the advent of PCR, definitive diagnosis of CNS viral infection was dependent upon either virus isolation from cerebrospinal fluid (CSF) or brain biopsy, or the demonstration of a virus specific intrathecal antibody response. Laboratory investigations such as viral culture although specific, lack sensitivity and frequently fail to provide results within a clinically useful period. ${ }^{4}$ Virus specific intrathecal antibody production, where studied in herpes simplex encephalitis, is reliably positive only 8-14 days after the onset of symptoms. ${ }^{56}$ Consequently, a positive result confirms the diagnosis only retrospectively.

Many CSF PCR assays lack validation because of the absence of accepted gold standards for the diagnosis of CNS infection. Two exceptions are the use of PCR to detect HSV in herpes simplex encephalitis or JC virus in progressive multifocal leucoencephalopathy, where brain biopsy has been used as the gold standard to determine assay sensitivity and specificity. ${ }^{78}$ However, the use of brain biopsy is limited by the invasive nature of the procedure.
Despite this lack of clinical validation, access to diagnostic CSF PCR assays is now widespread in the United Kingdom and elsewhere. Often these assays are developed in house. Although many are now standardised against independently produced proficiency panels, our understanding of the clinical interpretation of these PCR results remains incomplete. For instance, although Jeffery et al ${ }^{9}$ reported in their series that a patient with a positive PCR result was 88 times more likely to have a definite diagnosis of CNS viral infection than a patient with a negative PCR result, they commented that a negative result could be used with only moderate confidence to rule out CNS viral infection. Increasingly, positive PCR findings are reported in the CSF of patients where viral infection of the CNS is thought to be less likely, particularly in HIV seropositive individuals. ${ }^{10}$ Clinicians therefore often remain uncertain as to the significance of both negative and positive PCR findings.

In this study we employed multiplex PCR to detect a range of potentially neurotropic viruses as well as Toxoplasma gondii in CSF samples submitted to the diagnostic laboratory of a large London teaching hospital. Details of the patient's clinical presentation, investigations, and routine CSF examination were correlated with the PCR result. We used this approach to identify those factors that influence the likelihood of positive or negative PCR findings, and which thereby aid the clinician in the interpretation of PCR results.

Abbreviations: $\mathrm{Cl}$, confidence interval; $\mathrm{CMV}$, cytomegalovirus; $\mathrm{CNS}$, central nervous system; CSF, cerebrospinal fluid; EBV, Epstein Barr virus; $\mathrm{HHV}-6$, human herpes virus type 6; HSV-1 and 2, herpes simplex virus types 1 and 2; JCV, human polyomavirus JC; NPV, negative predictive value; PCR, polymerase chain reaction; PPV, positive predictive value; NS, not significant; VZV, varicella zoster virus; WCC, white cell count 


\section{METHODS}

Assays were carried out on stored CSF samples that were submitted to the Department of Infection at Guy's and St Thomas' Hospitals NHS Trust between April 1996 to May 2000. As routine CSF analysis was carried out irrespective of diagnosis in the same laboratory, all samples taken during this period were analysed. Samples were stored at $-70^{\circ} \mathrm{C}$. A sample volume of $100 \mu \mathrm{l}$ or more was the sole criteria for storing a CSF sample after completion of routine analysis. The CSF samples were tested in batches by multiplex PCR followed by reverse hybridisation to immobilised microbe specific oligonucleotide probes (line probe assay, LiPA) using a pre-release version of the CNS LiPA research kit (Innogenetics N.V., Gent, Belgium). This assay detected herpes simplex virus types 1 and 2 (HSV-l and 2), VZV, Epstein Barr virus (EBV), cytomegalovirus (CMV), human herpes virus type 6 (HHV-6), human enteroviruses, human polyomavirus JC (JCV), and the protozoan $T$ gondii with a sensitivity of approximately 100 genome equivalents per $100 \mu \mathrm{l} \mathrm{CSF}$. Assay performance was verified using external quality assessment panels for molecular detection of enteroviruses, HSV, and CMV from the European Union Quality Control Concerted Action. ${ }^{11}{ }^{12}$ The clinical data were obtained retrospectively by clinicians who were blinded to the CSF PCR result. Where hospital notes were available, detailed information was collected regarding the clinical episode associated with the CSF sample. The clinical findings at the time of presentation including: fever $\left(\geqslant 37.5^{\circ} \mathrm{C}\right)$, headache, meningism, seizures, rash, focal neurological signs, altered level of consciousness, immunosuppression, as well as the treating physician's discharge diagnosis were entered into a standardised proforma. Altered level of consciousness was defined as a Glasgow Coma Score $\leqslant 14$, or in infants the record of drowsiness. Patients were recorded as immunocompromised if they were one or more of the following: HIV seropositive, receiving chemotherapy, a transplant recipient, or were documented as having an inherited immunodeficiency. The time interval between the onset of neurological symptoms, such as meningism or paresis, and lumbar puncture was recorded.

The results of CNS imaging (computed tomography, magnetic resonance imaging, and in infants, cranial ultrasound) as well as electroencephalography (EEG) performed during the hospital admission were recorded as normal or abnormal. CSF white cell count (WCC), total protein, and culture results were recorded for each CSF sample. The WCC and protein levels were categorised as normal or abnormal using age related normal values. ${ }^{13}$

During the 4 year study period some patients presented to Guy's and St Thomas' Hospital on more than one occasion with different clinical conditions requiring CSF examination. These temporally separate presentations were defined as separate episodes. For other patients serial lumbar punctures were performed within one clinical episode. Where both detailed clinical as well as laboratory data were available, each clinical episode was classified as being either likely, possible, or unlikely to be a CNS viral infection (table 1) and this classification was related to the PCR result of the first CSF sampled in each episode. Analysis of the relationship between clinical or laboratory findings and the PCR result was performed using only the first CSF sample of the first clinical episode. The relationship between outcome and each clinical variable was initially analysed separately. A logistic regression model was then used to assess the relationship between the potentially explanatory variables and the PCR result, thereby allowing for confounding effects. As the distribution of the timing of lumbar puncture in relation to onset of neurological symptoms was skewed, the time delay was $\log$ transformed and points selected that split the sample data into four approximately equal groups. The groups thus defined were an interval of less then 3 days, 3-6 days, 7-13 days, or 14 days or more. The data were entered into a purpose designed Access database and subsequently analysed using Excel and GLIM. ${ }^{14}$ A p value of 0.05 or less was used to define statistical significance. Ethical approval for the study was obtained from the St Thomas' Hospital Ethics committee.

\section{RESULTS}

PCR results and cohort characteristics

A total of 787 CSF samples were obtained from 735 people who experienced 753 separate clinical episodes. Of these 735 people, $16(2 \%)$ had more than one clinical episode during the study period and $29(4 \%)$ had two or more CSF samples taken per clinical episode. Patients' ages ranged from newborn to 89 years. Of the 735 patients, 109 (15\%) were less than 1 year old, 189 (26\%) were between 1 and less than 16 years old, and 437 (59\%) were aged 16 years or older; 406 (55\%) of the patients were male.

Detailed clinical information from hospital records was available for $483 / 735$ (66\%) patients, corresponding to 494 clinical episodes. In this group of 483 patients, 73 (15\%) were less than 1 year old, 125 (26\%) were between 1 and less than 16 years old, $285(59 \%)$ were aged 16 years or older, 262 (54\%) were male, 61 (13\%) were known to be HIV seropositive, and 11 (2\%) were immunosuppressed through other causes. Thus age and gender for the whole group was very similar to the group for which clinical information was available.

Of the total 787 samples analysed, 97 (12\%) were PCR positive for one or more microbes and in $12(2 \%)$ multiple viruses were detected. In the group for which detailed clinical information was available, 59/494 (12\%) CSF samples were PCR positive. There was no significant difference in the proportion of samples that were PCR positive between age groups or gender. Table 2 details the specific PCR findings for the first CSF sample in each clinical episode both in the total cohort and the group with detailed clinical information. More than one virus detected in nine of the samples. Four of the five cases for which clinical information was available were HIV seropositive.

\section{PCR result compared to clinically defined categories}

A virus was detected in 32/108 (30\%) episodes where a CNS viral infection was thought clinically likely, whereas in the possible group 12/95 (12\%) CSF samples were positive. Amongst those episodes judged clinically unlikely to be due to a CNS viral infection, a virus was detected in only 15/291 $(5 \%)$. The clinical details related to these 15 samples are listed below. When the PCR findings for the likely group were compared to the possible and unlikely groups, the positive predictive value (PPV) was 54.2\% (confidence interval (CI) 40.75 to 67.28 ) and the negative predictive value (NPV) was $82.5 \%$ (CI 78.62 to 86.0 ). ${ }^{15}$ After exclusion of known HIV seropositive patients from the analysis, the PPV and NPV were $60.8 \%$ (CI 45.37 to 74.91 ) and $84.3 \%$ (CI 80.58 to 87.59 ), respectively. The PPV of detecting EBV by PCR was $8.3 \%$ (CI 0.21 to 38.48), whereas the PPV for detection of enteroviruses was $80.0 \%$ (CI 56.34 to 94.27 ).

A positive PCR result was found in 15 episodes where viral CNS infection was considered unlikely. An unequivocal alternative infectious, but non-viral, CNS disease was documented in four episodes (meningococcal meningitis in two patients, cryptococcal meningitis and leptospirosis in one case each). In three of these cases the virus detected was EBV and in the other it was CMV. A further three episodes occurred in infants, where an enterovirus, EBV, and HHV-6 were detected, respectively. In none of the cases was a 
Table 1 Classification of CNS viral infections using clinical and laboratory criteria*

Likely CNS viral infection (i and/or ii):

i. Raised CSF white cell count (defined by age group) accompanied by one or more of the following: meningism, headache, or fever ( $\geqslant 37.5^{\circ} \mathrm{C}$ ) (with no other explanation)

ii. Altered level of consciousness or focal neurological signs accompanied by fever $\left(\geqslant 37.5^{\circ} \mathrm{C}\right.$ ) or headache (with no other explanation)

Possible CNS viral infection:

Attending physician's final diagnosis of a viral CNS infection with any combination of signs or symptoms

Unlikely CNS viral infection (one of the following):

i. Another definite diagnosis (for example, multiple sclerosis or bacterial meningitis)

ii. Non-specific diagnosis (for example, febrile convulsion where the clinical or laboratory features were not consistent with the above categories)

iii. No definite diagnosis (for example, fever of unknown cause where the clinical or laboratory features were not consistent with the above categories)

*Classification adapted from Jeffery et al.

microbial cause for their septic illnesses identified at the time of admission. The remaining eight PCR positive CSF samples among this group were from patients with no clear evidence of CNS infection, although three were HIV positive.

\section{Clinical and laboratory findings}

The results of single variable analysis are shown in table 3. Statistically significant associations were noted between the detection of virus in CSF and the presence of meningism, headache, abnormal CSF WCC or protein level, and delay between onset of neurological symptoms and lumbar puncture. However, multivariable logistic regression revealed the association of abnormal CSF WCC to be significant only after omission of meningism, headache, and raised CSF protein level from the analysis. Furthermore, in the logistic regression model a significant association between focal neurological signs and failure to detect a virus by PCR was also found.

The relationship between the detection of a virus by PCR and the time interval between the onset of neurological symptoms and lumbar puncture was significantly non-linear (fig 1). Samples taken less than 3 days or more than 14 days into the disease process were less likely to be PCR positive than those taken between days 3 and 14. A similar statistically significant pattern was observed when the analysis was limited to those CSF samples that originated from patients thought likely to have CNS viral infections.

A normal CSF WCC was found in 31/59 (53\%) PCR positive CSF samples. Similar proportions of PCR positive CSF samples with normal WCC were found in both immunosuppressed and immunocompetent patients. Furthermore, 20/59 (34\%) PCR positive CSF samples had both normal WCC and protein levels. These samples were obtained from patients ranging in age from 7 weeks to 68 years, and 4/20 were from immunosuppressed patients. The PCR positive findings included: enterovirus (seven), HHV-6 (four), EBV (three),
HSV-1 (two), multiple viruses (two), CMV (one), and JC virus (one).

\section{DISCUSSION}

In this study multiplex PCR to detect a range of common neurotropic pathogens was performed on a large number of CSF samples from a wide age range of patients with a variety of clinical conditions. The study was set in a large teaching hospital providing secondary care to an inner city population as well as tertiary services in most paediatric and adult medicine specialties. Approximately 1 in 300 people is HIV seropositive in the hospital's catchment area. ${ }^{16}$

During the study period it was only possible to obtain clinical notes for $66 \%$ of the patients' episodes. It is possible that the collection of clinical data could have been biased towards patients who were hospitalised, thus over representing the seriously ill, and that a disproportionate number of patients whose notes were not found were those seen and then discharged from the Accident and Emergency department without admission. However, analysis of CSF report destinations revealed that overall $10 \%$ stated Accident and Emergency as a destination, compared with $11 \%$ in the cohort for which detailed information was available. In addition, the age and gender distribution, as well as PCR positivity rates in the group for which clinical information was available were very similar to those found in the whole group. Thus we believe a systematic bias in obtaining detailed clinical information is unlikely.

\section{The predictive value of a positive or negative PCR result}

Clinical assessment of the likelihood of a patient having a CNS viral infection was used in the absence of alternative gold standards to assess the PCR results. Virus was not detected in $70 \%$ of episodes where a CNS viral infection was considered likely, with a consequent NPV for the assay of

Table 2 PCR results for the first CSF sample obtained in each clinical episode

\begin{tabular}{|c|c|c|c|c|c|c|c|c|c|c|c|c|c|}
\hline & HSV-1 & HSV-2 & $\begin{array}{l}\text { HSV } \\
\text { untyped }\end{array}$ & VZV & CMV & EBV & HHV-6 & $\begin{array}{l}\text { Entero- } \\
\text { virus }\end{array}$ & JCV & $T$ gondii & $\begin{array}{l}\text { Multiple } \\
\text { viruses }\end{array}$ & $\begin{array}{l}\text { PCR } \\
\text { positive }\end{array}$ & $\begin{array}{l}\text { PCR } \\
\text { negative }\end{array}$ \\
\hline \multicolumn{14}{|l|}{$\begin{array}{l}\text { Group with detailed clinical } \\
\text { information }(n=494)\end{array}$} \\
\hline $\begin{array}{l}\text { Likely CNS viral infection } \\
\text { subgroup }(n=108)\end{array}$ & 0 & 6 & 1 & 1 & 1 & 1 & 2 & 16 & 1 & 0 & $3^{*}$ & 32 & 76 \\
\hline $\begin{array}{l}\text { Possible CNS viral infection } \\
\text { subgroup }(\mathrm{n}=95)\end{array}$ & 1 & 0 & 1 & 0 & 2 & 2 & 2 & 2 & 0 & 0 & $2 \dagger$ & 12 & 83 \\
\hline $\begin{array}{l}\text { Unlikely CNS viral infection } \\
\text { subgroup }(n=291)\end{array}$ & 2 & 0 & 0 & 0 & 1 & 9 & 1 & 2 & 0 & 0 & 0 & 15 & 276 \\
\hline $\begin{array}{l}\text { Group for which detailed clinical } \\
\text { information not available }(n=259)\end{array}$ & 4 & 2 & 0 & 2 & 2 & 6 & 4 & 3 & 1 & 1 & $4 \ddagger$ & 29 & 230 \\
\hline Total $(n=753)$ & 7 & 8 & 2 & 3 & 6 & 18 & 9 & 23 & 2 & 1 & 9 & 88 & 665 \\
\hline
\end{tabular}


Table 3 Proportion of CSF samples with normal or abnormal clinical or laboratory findings in which virus was detected by PCR

\begin{tabular}{|c|c|c|c|c|}
\hline Symptom, sign, or laboratory investigation* & Finding present $(\%)$ & Finding absent (\%) & Odds ratio $(95 \% \mathrm{Cl})$ & p Value \\
\hline Fever $\left(\geqslant 37.5^{\circ} \mathrm{C}\right)$ & $30 / 199(15 \%)$ & $21 / 224(9 \%)$ & $1.72(0.94$ to 3.15$)$ & NS \\
\hline Meningism & $14 / 75(19 \%)$ & $41 / 408(10 \%)$ & 2.05 (1.04 to 4.05$)$ & 0.0498 \\
\hline Headache & $27 / 160(17 \%)$ & $15 / 225(7 \%)$ & $2.84(1.44$ to 5.62$)$ & 0.0027 \\
\hline Rash & $8 / 50(16 \%)$ & $46 / 429(11 \%)$ & $1.59(0.69$ to 3.65$)$ & NS \\
\hline Focal neurology & $8 / 113(7 \%)$ & $47 / 370(13 \%)$ & $0.52(0.24$ to 1.16$)$ & NS \\
\hline Seizures & $13 / 127(10 \%)$ & $41 / 352(12 \%)$ & $0.86(0.44$ to 1.70$)$ & NS \\
\hline Encephalopathy & $14 / 139(10 \%)$ & $36 / 313(12 \%)$ & $0.86(0.44$ to 1.68$)$ & NS \\
\hline Immunosuppression & $11 / 71(16 \%)$ & $44 / 410(11 \%)$ & $1.53(0.74$ to 3.16$)$ & NS \\
\hline Abnormal CNS imaging & $14 / 161(9 \%)$ & $21 / 194(11 \%)$ & $0.78(0.38$ to 1.62$)$ & NS \\
\hline Abnormal EEG & $12 / 89(14 \%)$ & $4 / 20(20 \%)$ & $0.62(0.17$ to 2.24$)$ & NS \\
\hline Abnormal CSF WCC & $24 / 128(19 \%)$ & $29 / 344(8 \%)$ & $2.51(1.38$ to 4.55$)$ & 0.0028 \\
\hline Abnormal CSF protein & $23 / 132(17 \%)$ & $25 / 311(8 \%)$ & $2.41(1.30$ to 4.49$)$ & 0.0062 \\
\hline \multicolumn{5}{|l|}{$\begin{array}{l}\text { Delay between onset of symptoms and lumbar } \\
\text { puncturet }\end{array}$} \\
\hline$<3$ days & $6 / 101(6 \%)$ & & & \\
\hline 3 to $<7$ days & & $19 / 99(19 \%)$ & $0.270(0.10$ to 0.71$)$ & 0.0088 \\
\hline 7 to $<14$ days & & $15 / 90(16 \%)$ & $0.32(0.11$ to 0.87$)$ & 0.0329 \\
\hline$\geqslant 14$ days & & $2 / 92(2 \%)$ & $2.84(0.54$ to 14.94$)$ & NS \\
\hline
\end{tabular}

*Not all clinical or laboratory features were recorded for each patient; todds ratio calculated by comparison of findings in $<3$ days group with subsequent groups.

CSF, cerebrospinal fluid; EEG, electroencephalogram; NS, not significant; WCC, white cell count.

$82.5 \%$. Therefore, as reported by others, a negative result may only be used with moderate confidence to rule out a CNS viral infection. ${ }^{9}$ A variety of factors are likely to account for this finding. Firstly, the clinical categories were broadly defined. A wide spectrum of infectious and inflammatory diseases may mimic CNS viral infections, ${ }^{17}$ and the likely category required only the exclusion of diseases with other proven aetiologies, as opposed to corroborative evidence of CNS viral infection. Secondly, the assay sought nucleic acid for a limited number of viruses, and other microbes are associated with encephalitis in the United Kingdom. ${ }^{18}$ Two children in the series had encephalitic illnesses related to Mycoplasma pneumoniae infection (data not shown). Thirdly, as this study demonstrates, the time latency between the onset of neurological symptoms and CSF sampling influences the detection of viral nucleic acid. Amongst those patients thought likely to have had a CNS viral infection and where the latency was known, 17/102 (17\%) initial CSF samples were obtained at least 14 days after the onset of neurological symptoms, and none were PCR positive. Furthermore, 26 CSF samples were obtained less than 3 days after the onset of neurological symptoms, and only $12 \%$ of these were PCR

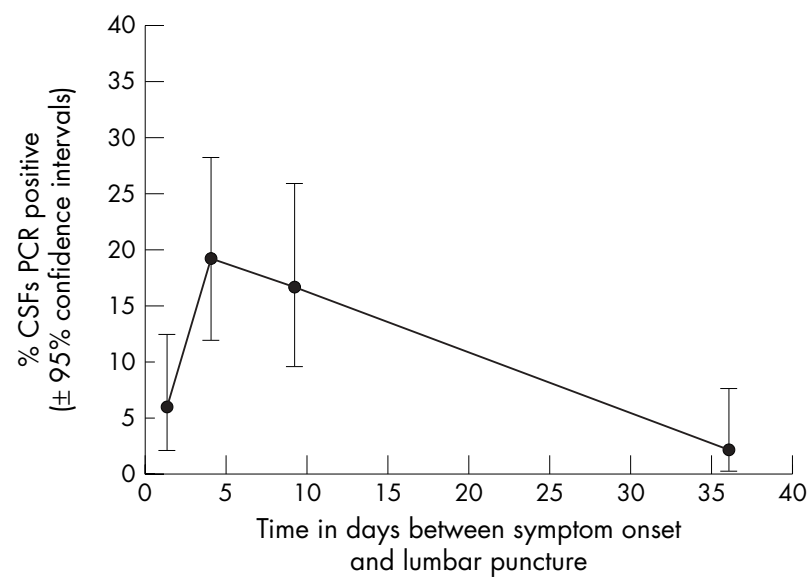

Figure 1 The relationship of virus detection by PCR with time delay between onset of neurological symptoms and lumbar puncture. *Numbers of PCR positive and negative CSF samples in each group are found in table 3. positive. Nonetheless, the $12 \%$ detection rate reported in this study is significantly higher than that reported by Jeffery et $a l^{9}(6.6 \%)$ and Tang et $a l^{19}(3.3 \%)$. This may reflect differences in assay sensitivity, in the range of microbes sought, differences in the patient populations under study, or differences in local policies relating to the timing of lumbar puncture.

The predictive value of a positive assay result was only 54\% as 27 positive PCR results were found in episodes classified as possible or unlikely CNS viral infections. In fact, $25 \%$ of positive PCR results were in patients thought unlikely to have CNS viral infections. These positive PCR findings may have occurred for a variety of reasons which include: laboratory contamination, atypical presentation of viral infection, dual aetiology amongst patients with other confirmed infections, or secondary viral reactivation in response to disease stress. Although PCR assays are susceptible to contamination, strict laboratory measures were taken to prevent this. ${ }^{20}$ Furthermore, the PCR findings were not random as the distribution of viruses found among the three clinical categories differed. Thus, EBV was predominantly detected in episodes where viral CNS infection was considered unlikely, whereas the majority of HSV-2 and enterovirus positive CSF samples were from episodes categorised as likely CNS viral infections.

Detection of EBV in CSF was initially reported to have high predictive value for primary CNS lymphoma in HIV patients with space occupying cerebral lesions, although a more recent study has documented a lower sensitivity value. ${ }^{21} 22$ The significance of detection of EBV in other settings is unclear, albeit that EBV PCR positivity may precede lymphoma onset in HIV patients. However, many of the patients in whom EBV was detected in the present study were not immunosuppressed. Positive EBV PCR results should therefore be interpreted with caution. Whilst in some cases detection of EBV might result from an unusual presentation of a CNS viral infection, in others it could represent an epiphenomenon. EBV might have been detected in CSF either through reactivation of the virus due to the concurrent stress to the host or through the latent infection of leucocytes trafficking into the intrathecal space. A similar leucocyte carriage hypothesis has been proposed to account for the detection by PCR of multiple viruses, but predominantly EBV and $\mathrm{CMV}$, in the synovial fluid and tissue of diseased joints. ${ }^{23}$ 
Three of the positive PCR results from episodes categorised as unlikely CNS viral infections occurred in infants. The signs of CNS viral infections are particularly difficult to ascertain in this age group. ${ }^{24}$ All suffered severe infections and lumbar puncture was performed as part of a screen for sepsis. In none of the cases was another organism identified to explain the infants' symptoms at the time of hospital admission. Thus the positive PCR results found in this study may well be indicative of the aetiological organism of their acute illnesses. It is increasingly recognised that enteroviruses, in particular, are a common cause of septic illness in infants, ${ }^{24}{ }^{25}$ and it is likely that other viruses will be proven to have a wider spectrum of clinical presentation in this age group.

One major reason for requesting virological investigation of CSF is to exclude a diagnosis of HSV encephalitis. Although no cases of classic HSV encephalitis occurred amongst the patients for whom we had detailed clinical information, HSV-1 was detected in a small number of patients. Mild or atypical cases have also been described by others. ${ }^{9}{ }^{26}{ }^{27}$ Where HSV-1 was detected in episodes classified as unlikely to be CNS viral infections, a pathogenic role for these agents cannot be entirely dismissed. In some cases, the significance of a positive PCR result that lies outside the described clinical phenotype might be corroborated by additional laboratory investigations. Evidence of a homologous virus specific intrathecal immune response could be sought, which if found would provide independent evidence of CNS infection. Alternatively other molecular biology techniques may be utilised. Quantitative CMV PCR has been used to distinguish productive infection from that due to the detection of latent CMV in CSF cells in patients with suspected CMV encephalitis. ${ }^{28}$ Similarly, the detection of mRNA specific for the lytic cycle of a DNA virus, and indicating productive infection, may also help distinguish latent from active infection. ${ }^{29}$ Our results indicate that virus can be detected in CSF when the probability of CNS viral infection is low. Utilisation of other laboratory techniques could help distinguish between a positive PCR result with high predictive value of disease aetiology from that with low predictive value for aetiology.

\section{Clinical and laboratory findings and PCR result}

The relationship between positive viral PCR and the interval between symptom onset and lumbar puncture showed that sampling early in the disease course ( $<3$ days) and delay ( 14 days or more) in sampling reduced the probability of detection. This finding was also observed when analysis was limited to patients categorised as likely to be suffering a CNS viral infection. Thus it is unlikely that the observation resulted from differences in patient populations between the four time groups. Whilst the relationship between time of symptom onset and lumbar puncture has been recognised in HSV encephalitis it has not been more widely reported for other CNS viral infections. ${ }^{30}$ Further study, ideally with serial CSF samples taken from individual patients, is required to define this diagnostic window more precisely and to determine if the timing of this window differs for patients with different clinical presentations or different infections. It has been suggested that the advent of rapid molecular diagnosis of viral CNS infections may allow the empirical use of antimicrobials drugs to be replaced with targeted therapy directed by PCR results. Our findings suggest that this is unlikely since antimicrobial therapy must be instituted as soon as possible to be of maximal benefit, and before the diagnostic window period identified here. We suggest that the role of PCR testing will be in guiding the continuation of antimicrobial therapy after the first few days, in determining the need for further investigations, and in prognostication.

Both single and multivariable analysis showed that a raised CSF WCC was associated with the detection of virus by
PCR in CSF. However, the majority of PCR positive CSF samples had no pleocytosis and in many cases neither pleocytosis nor elevated CSF protein level (34\%). This finding applied both to PCR positive samples from episodes classified as likely CNS viral infection as well as those classified unlikely or possible. Although a similar finding has been observed, ${ }^{31}$ others have argued that CSF samples with neither pleocytosis nor raised protein level should be excluded from PCR testing for viruses. ${ }^{19}$ Our findings do not support such an approach. CSF pleocytosis is a better indicator of meningeal than brain parenchymal inflammation. Consequently a greater proportion of patients suffering viral encephalitis, as opposed to viral meningitis, have a normal CSF WCC despite suffering a worse outcome. ${ }^{32} 33$ The diagnostic yield of PCR investigations should be maximised by using sensitive assays to detect pathogens in appropriately timed CSF samples, rather than by applying selection criteria based upon laboratory CSF parameters.

\section{ACKNOWLEDGEMENTS}

We thank Spencer Wilkinson, Department of Infection, Guy's and St Thomas' Trust for assistance with database construction. We also thank Helen Dunn, Diane Cronin, and other staff members of the Department of Infection, Guy's and St Thomas' Trust who helped to facilitate this study.

\section{Authors' affiliations}

N W S Davies, A V Swan, M K Sharief, Department of Clinical

Neurosciences, King's College London, London, UK

L J Brown, D Irish, J Banatvala, Department of Infection, Guy's and St Thomas' Hospitals NHS Trust, London, UK

L J Brown, J Banatvala, P Muir, Department of Infectious Diseases, King's College London, London, UK

J Gonde, R O Robinson, Department of Paediatric Neurology, Guy's and St Thomas' Hospitals NHS Trust, London, UK

R S Howard, Department of Neurology, Guy's and St Thomas' Hospitals NHS Trust, London, UK

NWSD was supported through a Personal Training Award from the Charitable Foundation of Guy's and St Thomas' Hospitals. UB was supported by a Research Contract with Innogenetics N.V., Gent, Belgium.

Competing interests: Peter Muir received funds from Immogenisation N.V., Gent, Belgium to develop CSF testing for the diagnosis of CNS infections. None of the other authors has competing interests to declare.

*Current address: Department of Microbiology, Frimley Park NHS Trust, Surrey, UK

${ }^{* *}$ Current address: Health Protection Agency South West, Bristol, UK

\section{REFERENCES}

1 DeBiasi RL, Tyler KL. Polymerase chain reaction in the diagnosis and management of central nervous system infections. Arch Neurol 1999;56:1215-9.

2 Bergstrom T. Polymerase chain reaction for diagnosis of varicella zoster virus central nervous system infections without skin manifestations. Scand J Infect Dis Suppl 1996; 100:41-5.

3 Schlesinger $Y$, Tebas P, Gaudreault-Keener M, et al. Herpes simplex virus type 2 meningitis in the absence of genital lesions: improved recognition with use of the polymerase chain reaction. Clin Infect Dis 1995;20:842-8.

4 Linde A, Klapper PE, Monteyne P, et al. Specific diagnostic methods for herpesvirus infections of the central nervous system: a consensus review by the European Union Concerted Action on Virus Meningitis and Encephalitis. Clin Diagn Virol 1997;8:83-104.

5 Skoldenberg B, Forsgren M, Alestig K, et al. Acyclovir versus vidarabine in herpes simplex encephalitis. Randomised multicentre study in consecutive Swedish patients. Lancet 1984;2:707-11

6 Kahlon J, Chatterjee S, Lakeman FD, et al. Detection of antibodies to herpes simplex virus in the cerebrospinal fluid of patients with herpes simplex encephalitis. J Infect Dis 1987; 155:38-44.

7 Weber T, Turner RW, Frye S, et al. Specific diagnosis of progressive multifocal leukoencephalopathy by polymerase chain reaction. J Infect Dis 1994; 169:1138-41

8 Lakeman FD, Whitley RJ. Diagnosis of herpes simplex encephalitis: application of polymerase chain reaction to cerebrospinal fluid from brainbiopsied patients and correlation with disease. National Institute of Allergy and Infectious Diseases Collaborative Antiviral Study Group. J Infect Dis $1995 ; 171: 857-623$. 
9 Jeffery KJ, Read SJ, Peto TE, et al. Diagnosis of viral infections of the central nervous system: clinical interpretation of PCR results. Lancet 1997;349:313-7.

10 Quereda C, Corral I, Laguna F, et al. Diagnostic utility of a multiplex herpesvirus PCR assay performed with cerebrospinal fluid from human immunodeficiency virus-infected patients with neurological disorders. J Clin Microbiol 2000;38:3061-7.

11 Schloss L, van Loon AM, Cinque P, et al. An international external quality assessment of nucleic acid amplification of herpes simplex virus. J Clin Virol 2003;28:175-85.

12 van Vliet KE, Muir P, Echevarria JM, et al. Multicenter proficiency testing of nucleic acid amplification methods for the detection of enteroviruses. J Clin Microbiol 2001;39:3390-2.

13 Campbell AGM, Mclntosh N. Forfar and Arneil's textbook of paediatrics. Edinburgh: Churchill Livingstone, 1992:1976-7.

14 Francis B, Green M, Payne C (eds). GLIM 4 - The statistical system for generalized linear interactive modelling. Oxford: Clarendon Press, 1993.

15 Armitage P, Berry G, Matthews JNS. Statistical methods in medical research Oxford: Blackwell Science, 2002:119.

16 CDSC. SOPHID update for 2000: prevalent diagnosed infections in England, Wales and Northern Ireland. Commun Dis Rep Wkly 2002;12:7-13.

17 Whitley RJ, Cobbs CG, Alford CA Jr, et al. Diseases that mimic herpes simplex encephalitis. Diagnosis, presentation, and outcome. NIAD Collaborative Antiviral Study Group. JAMA 1989;262:234-9.

18 Kennedy CR, Chrzanowska K, Robinson RO, et al. A major role for viruses in acute childhood encephalopathy. Lancet 1986;1:989-91.

19 Tang YW, Hibbs JR, Tau KR, et al. Effective use of polymerase chain reaction for diagnosis of central nervous system infections. Clin Infect Dis 1999:29:803-6.

20 Kwok S, Higuchi R. Avoiding false positives with PCR. Nature 1989;339:237-8.

21 Cinque $P$, Brytting $M$, Vago $L$, et al. Epstein-Barr virus DNA in cerebrospinal fluid from patients with AIDS-related primary lymphoma of the central nervous system. Lancet 1993;342:398-401.

22 Ivers LC, Kim AY, Sax PE. Predictive value of polymerase chain reaction of cerebrospinal fluid for detection of Epstein-Barr virus to establish the diagnosis of HIV-related primary central nervous system lymphoma. Clin Infect Dis 2004;38:1629-32.
23 Stahl HD, Hubner B, Seidl B, et al. Detection of multiple viral DNA species in synovial tissue and fluid of patients with early arthritis. Ann Rheum Dis 2000;59:342-6.

24 Rorabaugh ML, Berlin LE, Heldrich F, et al. Aseptic meningitis in infants younger than 2 years of age: acute illness and neurologic complications. Pediatrics 1993;92:206-11.

25 Verboon-Maciolek MA, Nijhuis M, van Loon AM, et al. Diagnosis of enterovirus infection in the first 2 months of life by real-time polymerase chain reaction. Clin Infect Dis 2003;37:1-6.

26 Fodor PA, Levin MJ, Weinberg A, et al. Atypical herpes simplex virus encephalitis diagnosed by PCR amplification of viral DNA from CSF. Neurology 1998;51:554-9.

27 Domingues RB, Tsanaclis AM, Pannuti CS, et al. Evaluation of the range of clinical presentations of herpes simplex encephalitis by using polymerase chain reaction assay of cerebrospinal fluid samples. Clin Infect Dis 1997;25:86-91.

28 Wildemann B, Haas J, Lynen N, et al. Diagnosis of cytomegalovirus encephalitis in patients with AIDS by quantitation of cytomegalovirus genomes in cells of cerebrospinal fluid. Neurology 1998;50:693-7.

29 Weinberg A, Li S, Palmer M, et al. Quantitative CSF PCR in Epstein-Barr virus infections of the central nervous system. Ann Neurol 2002;52:543-8.

30 Cinque $\mathbf{P}$, Cleator GM, Weber T, et al. The role of laboratory investigation in the diagnosis and management of patients with suspected herpes simplex encephalitis: a consensus report. The EU Concerted Action on Virus Meningitis and Encephalitis. J Neurol Neurosurg Psychiatry 1996;61:339-45.

31 Minjolle S, Arvieux C, Gautier AL, et al. Detection of herpesvirus genomes by polymerase chain reaction in cerebrospinal fluid and clinical findings. J Clin Virol 2002;25(suppl 1):S59-70.

32 Simko JP, Caliendo AM, Hogle K, et al. Differences in laboratory findings for cerebrospinal fluid specimens obtained from patients with meningitis or encephalitis due to herpes simplex virus (HSV) documented by detection of HSV DNA. Clin Infect Dis 2002;35:414-9.

33 Andersson SO, Biorksten B, Burman LA. A comparative study of meningoencephalitis epidemics caused by echovirus type 7 and coxsackievirus type B5. Clinical and virological observations during two epidemics in northern Sweden. Scand J Infect Dis 1975;7:233-7. 